\title{
TIME FUNCTIONS APPROPRIATE FOR NUCLEAR EXPLOSIONS
}

\author{
By L. J. Burdick AND D. V. HeLmberger
}

\begin{abstract}
The source-time function of megaton class nuclear explosions has been determined by modeling teleseismic short- and long-period body waves with synthetic seismograms. A simple analytic expression for the time function was used to closely match observations from both Novaya Zemlya and the U.S. test site at Amchitka. It was found that the time functions of all the events have a substantial overshoot. It was also found that, although the durations of the time functions did appear to depend on yield, the effect was very difficult to observe even in short-period records. All synthetics were computed by assuming a simple point source in a layered elastic half-space. It was not necessary to appeal to any nonlinear processes in the source region to explain the observations. Numerical calculations are presented to show that tectonic release triggered by earthquakes does not have a substantial effect on the $\boldsymbol{P}$ waves unless the longperiod level of the tectonic event is as large or larger than the long-period level of the explosion. The $p S$ wave, on the other hand, is shown to be very sensitive to even a moderate amount of tectonic release.
\end{abstract}

\section{INTRODUCTION}

The complex physical processes that occur in the vicinity of a buried nuclear explosion are difficult to model even with sophisticated numerical codes. The seismic waves which are radiated to large distances on the other hand can be easily modeled with a simple point source. In this investigation, we have attempted to find pointsource models for teleseismic $P$-wave observations of megaton class events from both Novaya Zemlya and Amchitka. These point-source models will serve at least three important purposes. First, they can be used to constrain the equivalent farfield behavior of the large code calculations. Second, they would aid in solving the discrimination problems if the $M_{S}-m_{b}$ criterion was not decisive and, finally, they would permit more extensive use of explosion data in wave form modeling studies of the Earth's velocity structure (Helmberger and Wiggins, 1971; Helmberger, 1973). Analytic forms for the source-time history of an explosive point source have been proposed by Toksöz et al. (1964), Haskell (1967), Mueller and Murphy (1971), and von Seggern and Blandford (1972). These models were originally based on or have since been tested against surface-wave spectra and both close in and teleseismic body-wave spectra (Tsai and Aki, 1971; Molnar, 1971; Aki et al., 1974). In this investigation, the predictions of a point-source model are compared to teleseismic body-wave forms in the time domain. Both long-period and short-period observations are used to constrain the source model over a relatively broad period range. A large new body of teleseismic data has become available for use in this study because of the extensive nuclear testing program which has recently been carried out in the Soviet Union. These data combined with observations of the largest Amchitka blast, Cannikin, provide important new restrictions on the class of allowable source models.

\section{A Point-Source Model}

The explosive sources will be modeled as simple point dilatations in all of the calculations to be presented here. It will also be assumed that linear elasticity and plane layered velocity structures can be used to describe wave propagation in the 
source region. More sophisticated models are for the most part unwarranted since the data consist exclusively of teleseismic body waves. Those small inadequacies of point dilatation models which do become apparent can still be interpreted at least qualitatively in terms of tectonic strain release or nonlinear processes in the source region. Under the point source approximation, it is only necessary to specify a time function, the depth, and a plane-layered velocity structure for the source region in order to compute synthetics. The depth and wave velocities play a very significant role because they control the size and arrival time of the $p P$ phase which generally has just as much influence on the wave form as direct $P$. An important goal of this report will be to establish the relative importance of the shape of the time function as opposed to the free surface effect in determining the wave form.

The time function parameterization which will be used in the synthetic seismogram calculations is the one proposed by von Seggern and Blandford (1972). At teleseismic distances, it predicts pulse shapes which are for all practical purposes equivalent to those predicted by the models of Haskell (1967) or Mueller and Murphy (1971), but it is mathematically much simpler. An alternative might have been the source model of Toksöz et al. (1964) or Helmberger and Harkrider (1972), but these models have the disadvantage that they cannot be used to describe steplike source functions. Some dc component should realistically be expected if a cavity is formed by the explosion. They also predict far-field, body-wave time functions which are singular at time equal zero. The von Seggern and Blandford pulse can be either a near-step or a near-impulse. The reduced displacement potential for this model is written

$$
\psi(t)=\psi(\infty)\left[1-e^{-k t}\left(1+k t-B(k t)^{2}\right)\right] H(t)
$$

$k$ and $B$ are the adjustable parameters of the model and $\psi(\infty)$ is the dc source strength given by

$$
\psi(\infty)=\frac{r_{e l}^{3} P_{0}}{4 \mu}
$$

$r_{e l}$ is the elastic radius at the source, $P_{0}$ is the steady-state cavity pressure, and $\mu$ is the shear modulus. The far-field displacement pulse is then just the time derivative of $\psi(t)$.

The data set contains wave forms only from epicentral distances between $30^{\circ}$ and $80^{\circ}$. This means that a first-motion approximation is valid for describing wave propagation near the source and that the wave forms are undistorted by velocity structure near their turning point. The synthetic seismogram for the direct $P$ wave is written

$$
S(t)=A\left[I(t) * Q(t) * \frac{d}{d t} \psi(t) * C(t)\right]
$$

$I(t)$ and $Q(t)$ are the instrument and Futterman $Q$ filter, * is the convolution operator, $C(t)$ is the response of the source crust, and $A$ is a scaling coefficient for geometrical spreading and the receiver function (Langston and Helmberger, 1975). The value of $t^{*}$ in the Futterman operator was set a 1.0 for the $P$-wave calculations (Anderson and Hart, 1978). Butler (in preparation) has recently completed a study of the variations of $t^{*}$ across the U.S. using Novaya Zemlya data and has found that generally $t^{*}=1.0 \pm 0.2$. The operator $C(t)$ can be computed either by using 
geometric ray theory or by using propagator matrices (Fuchs, 1966). The former approach is convenient for half-space calculations since only two rays are involved, and the latter is convenient for multilayered calculations since all internal multiples are automatically included. Both approaches will be used in the calculations to follow. It is also useful to define an effective source function

$$
S(t)=\frac{d}{d t} \psi(t) * C(t)
$$

which contains both the effects of the source-time function and the source crust. This composite operator gives the pulse shape which is ultimately radiated to the far field. Equation (3) generalizes immediately to the case of teleseismic $p S$ waves with the substitution of the appropriate crustal response and scaling coefficient. Tectonic release can be modeled by simply adding in the response of the crust to a buried double couple. We have used this formalism to compute synthetic wave forms and to match them with data by trial and error. We will now discuss the allowed ranges for the time-function parameters which were established. We will also cover the relative importances of the time function, free surface interaction, and tectonic release in determining the wave form.

\section{$P$ Waves From Novaya Zemlya and Amchitka}

WWSSN stations in the U.S. are almost all situated within a narrow band of epicentral ranges between $65^{\circ}$ and $75^{\circ}$ from the Soviet nuclear test site at Novaya Zemlya. They all lie between $45^{\circ}$ and $70^{\circ}$ from the U.S. site at Amchitka. Their location combined with their high density and relatively good transparency makes them ideal for source studies of explosions from the two sites. We shall rely on data from U.S. stations throughout this discussion. In recent years, a large number of devices ranging in yield from 0.3 to $5 \mathrm{Mt}$ [all yields quoted in this report are from Dahlman and Israelson (1977)] have been exploded, providing a sizeable body of wave-form data. Good quality records are available from both the short-period $(\sim 1$ sec) and the long-period $(\approx 15 \mathrm{sec})$ WWSSN instruments. The Novaya Zemlya data set has one major drawback in that only a few of the moderate sized events $(\sim 1.5$ Mt) recorded well on both instruments. Because of the standard WWSSN gain settings, small events record well on the short periods but are too small on the long periods. Large explosions record well on the long periods but do not produce good wave forms on the short. The light pen begins to skip and skewing becomes a serious problem. Sometimes it is possible to obtain reliable amplitude estimates even if the wave form is poor so that the amplitude data set is relatively larger than the waveform data set. So far as the wave-form data from Novaya Zemlya is concerned, the only possible approach is to determine a shallow source model which fits the shortperiod records from small events and a separate deep-source model to fit the long periods from large events. The WWSSN gains were specially set to appropriate levels for the Cannikin blast. Therefore, there is good wave-form and amplitude data from both instruments which can be used to further constrain the source model.

A source model for Novaya Zemlya events. We begin by modeling one of the moderate sized Soviet events which did record well on both instruments. Of the U.S. stations, one of the most transparent is at Atlanta, Georgia. Short-period records of nuclear events always appear there as a single simple pulse. The records of the November 2, 1974 event are of sufficiently good quality that the digitized signals can be treated as an analytic time series. The effective sources, $S(t)$ from equation (4) can be determined by a simultaneous short period-long period decon- 
volution procedure originally introduced by Burdick and Mellman (1976). In this procedure, the theoretical instrument and $Q$ operator are deconvolved from each of the two seismograms. The resulting time series are averaged in frequency domain using a weighting function which mirrors the instrument sensitivities. The resulting spectrum is filtered lightly with a Gaussian filter and transformed back to time domain. The simultaneous deconvolution result for the ATL records is shown at the top of Figure 1. The effective source shape consists of a positive direct $P$ pulse, a negative $p P$ pulse, and very little else. The remaining portion of the signal is quite small. This indicates that the source-time function and crustal response are actually quite simple and that they can be easily modeled. Several observed long- and shortperiod ATL records including those used in the deconvolution are shown under the deconvolved pulse. At the bottom of the figure are synthetics computed by convolv-

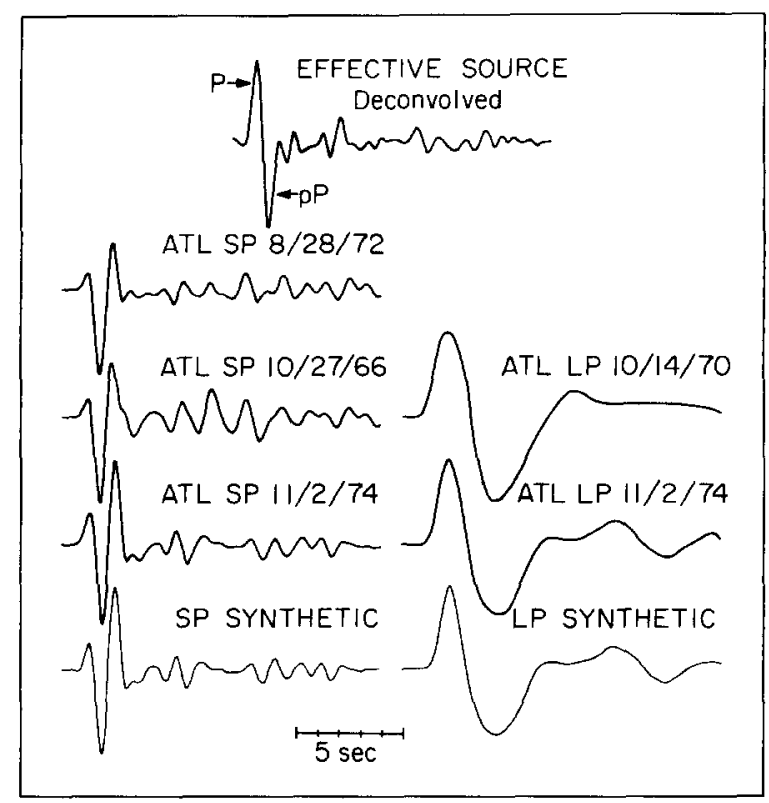

FIG. 1. The effective source at the top was determined by the simultaneous deconvolution procedure. Short-period data on the left and long-period data on the right are compared with synthetics computed by reconvolving the source with the attenuation and instrument operators.

ing the source function with the instrument and $Q$ operators. The good agreement between the synthetic and observed seismograms shows that the deconvolved pulse is a good broad-band estimate of the effective source pulse. Fitting the deconvolved pulse is relatively simple compared to attempting to find a model which fits two separate seismograms at once by trial and error.

One surprising feature of the effective source is that the $P$ pulse appears to be somewhat smaller than the $p P$ pulse. The free surface reflection coefficient should be about 0.8 or 0.9 at these ranges. However, this effect can be easily modeled by using a source-time function with the proper amount of overshoot. This corresponds to finding an appropriate value for $B$ in equation (1). Figure 2 compares theoretical effective source pulses to the observed for various amounts of overshoot in the source. On the left of the figure are reduced displacement potentials, $\psi(t)$, for several values of $B$. Increasing $B$ increases the amount of overshoot. The far-field pulse is $(d / d t) \psi(t)$ so, if $\psi(t)$ overshoots, the far-field pulse has a negative part. Constructive interference between the negative part of the $P$ arrival and $p P$ causes the $p P$ phase 
to appear larger than $P$. The deconvolved pulse can be best explained if the reduced displacement potential long-time level is $\frac{1}{3}$ to $\frac{2}{3}$ of the maximum value or $2<B<7$. For the calculations in Figure 2 the value of $k$ was fixed at $5 \mathrm{sec}^{-1}$. Figure 3 illustrates that the value of $k$ could vary from 4 to $9 \mathrm{sec}^{-1}$ without seriously affecting the fit. If

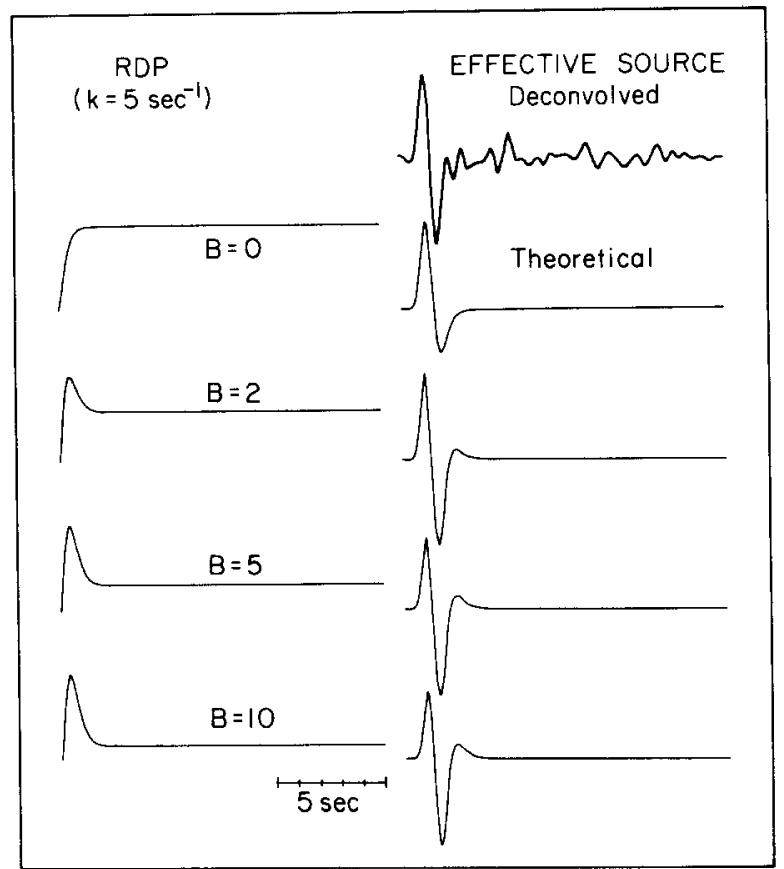

FIG. 2. Theoretical effective sources are compared to the deconvolved result for a range of values of the overshoot parameter, $B$. The reduced displacement potentials (RDP) are shown on the left. The best agreement occurs for a $B$ of roughly 5 .

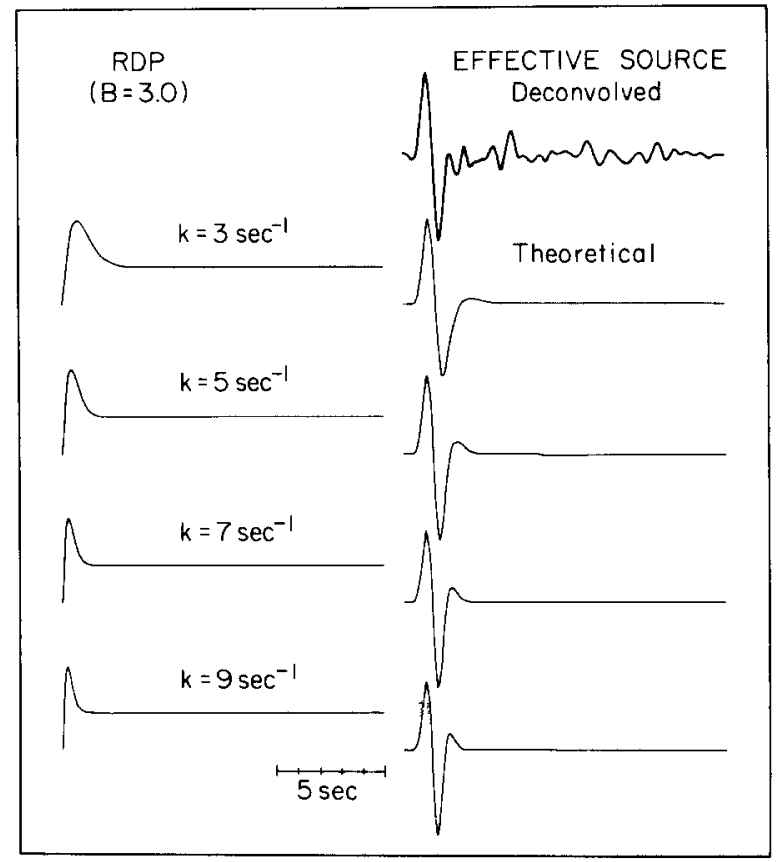

FIG. 3. Theoretical effective sources are compared to the deconvolved for a range of values of the time scaling parameter. The reduced displacement potentials (RDP) are shown on the left. The best agreement occurs for a $k$ of roughly $5 \mathrm{sec}^{-1}$. 
the value of $k$ is less than 4 , the width of the theoretical pulse becomes too large and if it is larger than 9 , the interference effect of the $p P$ and the overshoot becomes hard to model. The limits on $k$ are very dependent on our choice of the value for $t^{*}$. Assuming that $t^{*}$ was smaller would result in substantially lower estimates of $k$.

The calculations in Figures 2 and 3 were made by assuming that the source was buried in a half-space. The delay time for the $p P$ phase, $T_{p P}$, was set at $0.6 \mathrm{sec}$. The results of the calculation depended very strongly on this choice. We shall now demonstrate that the depth of burial of the source is the most important factor in determining the wave shape. As explained previously, most of the short-period data for Novaya Zemlya comes from small bombs and the long-period data from larger

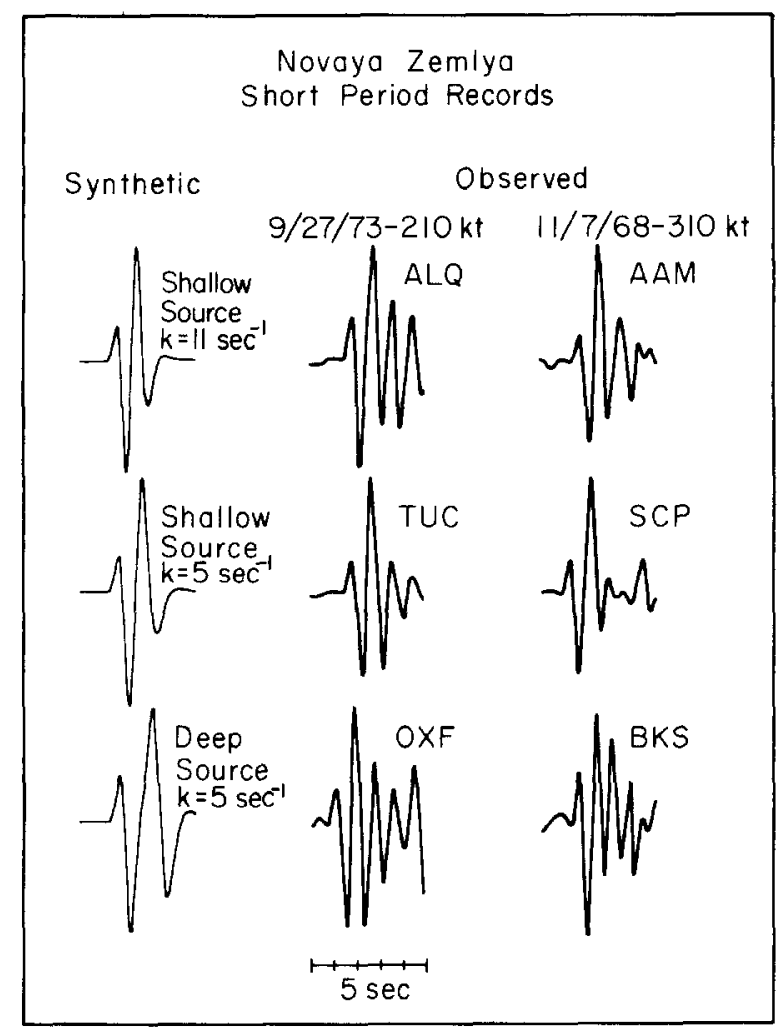

FIG. 4. Synthetic short-period records are compared to observed Novaya Zemlya records. A best fit with $k=11 \mathrm{sec}^{-1}$ and $B=7$ is shown at the top left $k$ is reduced to $5 \mathrm{sec}^{-1}$ for the middle synthetic which has a very small effect. The depth is increased for the bottom synthetic which has a dramatic effect.

bombs. Since the depth of burial increases with yield, it is reasonable to expect that a deep source should fit the long-period data and a shallow source should fit the short. Figure 4 shows a group of observed short-period records from two small events. At the top left of the figure is a synthetic for a source function which fits quite well. The source is shallow $\left(T_{p P}=0.4 \mathrm{sec}\right)$ and the time function parameters are $k=11 \mathrm{sec}^{-1}$ and $B=7$. Next is a synthetic with $k=5 \mathrm{sec}^{-1}$ which does not quite fit as well, but the difference is very small. The synthetic at the bottom left was computed for $k=5 \mathrm{sec}^{-1}, B=7$, and a deep source $\left(T_{p P}=1.0\right)$. The effect is very large indicating that it is very difficult to resolve changes in the source parameter $k$ but easy to resolve source depth. A similar comparison for long-period observations 
is presented in Figure 5. The best fit is for the deep source with $k=3 \mathrm{sec}^{-1}$ and $B$ $=7$, but there is only a slight change if $k$ is increased to $5 \mathrm{sec}^{-1}$. The shallow source very clearly does not fit the data. One feature of the source function that is required is a substantial overshoot. All of the observed long-period Novaya Zemlya records have a large second peak (peak $B$ in Figure 5). This effect cannot be produced synthetically unless the overshoot is present. It enhances the negative $p P$ arrival as discussed previously which enlarges the second peak of the seismogram. The longperiod synthetics do not fit the duration of peak $B$ as well as might have been desired. However, examination of the observed records shows that this duration is not very consistent between stations. Peak $B$ also shows some fine structure. We will demonstrate in the following sections that these effects could be due to tectonic release.

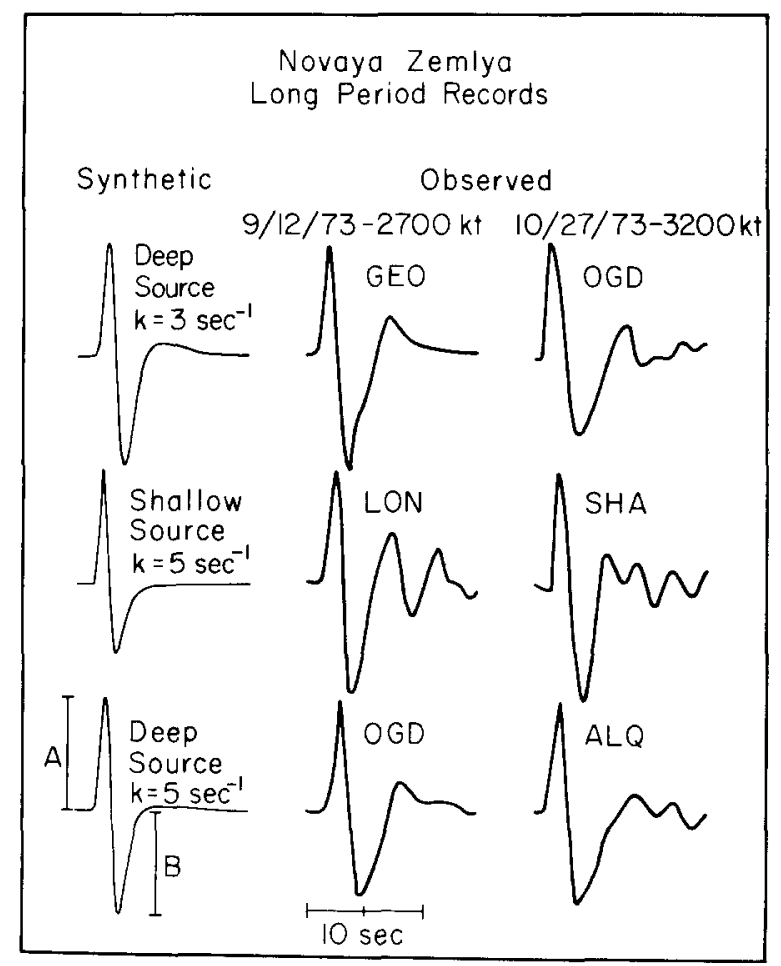

FIG. 5. Synthetic long-period records are compared to observed Novaya Zemlya records. A best fit with $k=3 \mathrm{sec}^{-1}$ and $B=7$ is shown at the top left; $k$ is increased to $5 \mathrm{sec}^{-1}$ for the bottom synthetic which has little effect. The depth is decreased for the middle synthetic which has a dramatic effect.

A source model for Cannikin. The body waves from the Amchitka test, Cannikin, provide an interesting cross-check on the model developed for Novaya Zemlya. The depth of the source and some of the details of the source crust are known independently, permitting better resolution of the time function. The Cannikin explosion has already been the object of extensive analysis (Engdahl, 1972; Toksöz and Kehrer, 1972; Willis et al., 1972; Bakun and Johnson, 1973). Nonetheless, there is still some important new information which can be gained through wave-form analysis of the teleseismic body waves.

The depth of the explosion was $1791 \mathrm{~m}$ (Toksöz and Kehrer, 1972). Engdahl (1972) determined a layered velocity model for the source region from travel-time and well-log data. The teleseismic response for this rather detailed crustal model 
can be easily computed with the matrix propagator technique of Harkrider (1964) and Fuchs (1966). Figure 6 compares the synthetics for an explosion in the Engdahl model (crust I) to observed short-period records of Cannikin. The synthetics do not fit the small shoulder in the second positive peak of the data. This feature, though small, is significant because it is consistent in all the data and because it occurs early in the wave form where the records are generally most reliable. Some minor trial and error perturbations quickly show that the feature is the $p P$ phase arriving later than predicted. To achieve this effect, the $P$-wave velocity in the top layers of

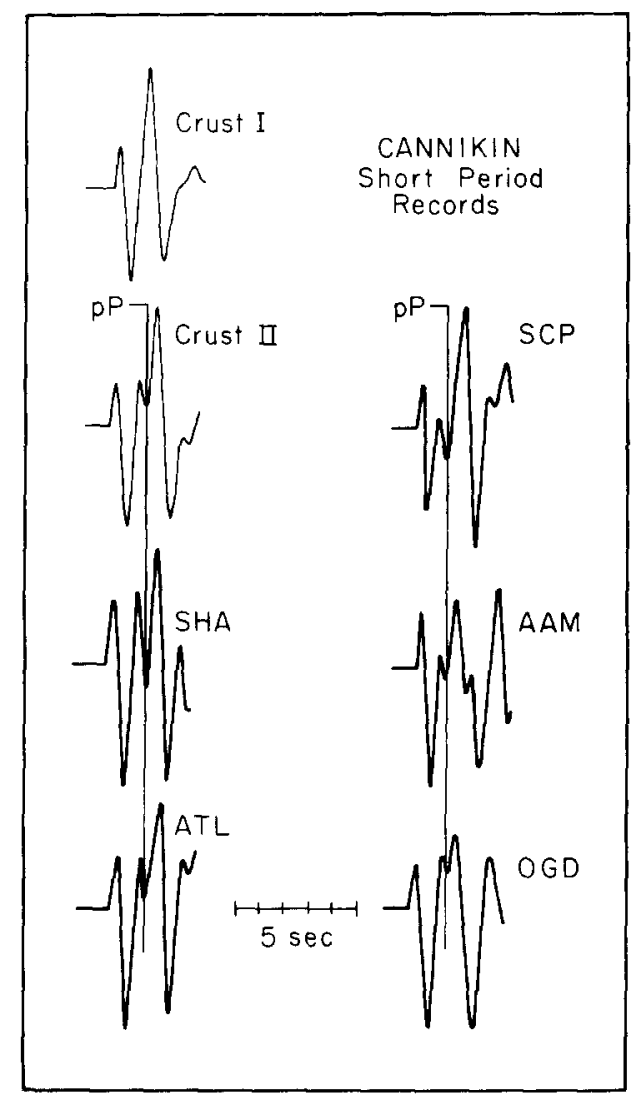

FIG. 6. Synthetic short-period wave forms for two different crust models are compared to observed Cannikin records. Crust model II more closely predicts the arrival time of $p P$. The time function parameters are $B=2$ and $k=5 \mathrm{sec}^{-1}$.

the crustal model has to be reduced by 25 per cent to an average value of $2.9 \mathrm{~km} /$ sec. A synthetic for this slower model (crust II) is also shown in Figure 6. It agrees very closely with the observed records. Figure 7 compares long-period synthetics for models I and II with observed records. Model II again appears to fit better. The structure in the second peak of the records is caused by a reverberation in the slow layers at the top of the source crust. This type of interaction helps to explain the variations in the shape of the second peak from source to source. The time function parameters used for the synthetic calculations were $B=2$ and $k=5$.

There are two very significant results which have emerged from modeling the Cannikin data. The first is that because of the depth of Cannikin the $P$ phase moves forward almost clear of the $p P$ phase on the short-period record. The point-source 
synthetic seismograms predict this effect almost perfectly, which indicates that the associated approximations are valid. The second is that this clear $P$ wave can be modeled with a time function which is very similar to the time function of the small Novaya Zemlya events (see Figure 4). The amount of overshoot is somewhat reduced, but the apparent duration of the $P$-wave arrival is about the same. This implies that within the resolution capabilities of the WWSSN instrument, a 5-Mt blast would be very difficult to discriminate from a $0.3-\mathrm{Mt}$ blast on the basis of the shape of the time function. There are only two major changes which can be observed as the yield of the bomb increases over this range. This first is the increase of the amplitude due to an increase in cavity radius [see equations (1) and (2)], and the second is the change in the interference pattern of $P$ and $p P$ due to the increase of the source depth.

The need to decrease the velocities of the top layers of the Engdahl crust model

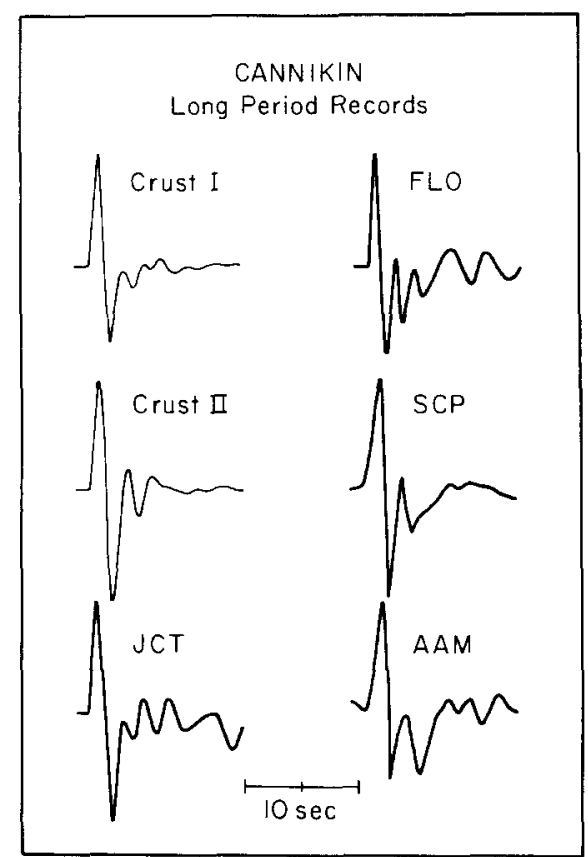

FIG. 7. Synthetic long-period wave forms for two different crust models are compared to observed Cannikin records. Crust model II fits slightly better. The time function parameters are $B=2$ and $k=5$ $\sec ^{-1}$.

may simply have been due to the inaccuracy of the model. Alternatively, this could be an effect of the bomb itself. The two-way travel time of the $p P$ phase through the melted and pulverized rock in the source region may be significantly larger than in the normal crust of the region. This possibility was discussed by Bakun and Johnson (1973). They determined the crustal response $C(t)$ for the Cannikin source region using a homomorphic deconvolution procedure on LRSM short-period data. Figure 8 compares their deconvolved impulse train for HNME with the impulse train computed with propagator matrices for crust model II. Both results show the same large time separation between $P$ and $p P$. Bakun and Johnson concluded that a third arrival was present which they termed a "slapdown phase" (Frasier, 1972; Springer, 1974). Figure 8 shows that it looks much like the crustal reverberations, but is about twice the size. Figure 6 clearly shows that the short-period data can be fit without this arrival, so we conclude that either the homomorphic deconvolution did not 
completely separate the source from the crust response or there was some more fundamental difference between the LRSM and WWSSN data.

Amplitude Data. Absolute amplitude data from explosions is difficult to interpret because it depends on so many unknown factors. Equations (1) and (2) show that these include the dc source strength $\psi(\infty)$, the source depth, the shape or frequency content of the source function, the amount of attenuation, and geometric spreading. The passband of the seismograph also has an effect, but the instrument characteristics are generally well known. The amplitude ratio of the short- to long-period wave form is easier to deal with since it depends only on the source depth, source shape, and attenuation. The average short- and long-period amplitudes at WWSSN stations in the U.S. were measured for 10 Novaya Zemlya events plus Cannikin.

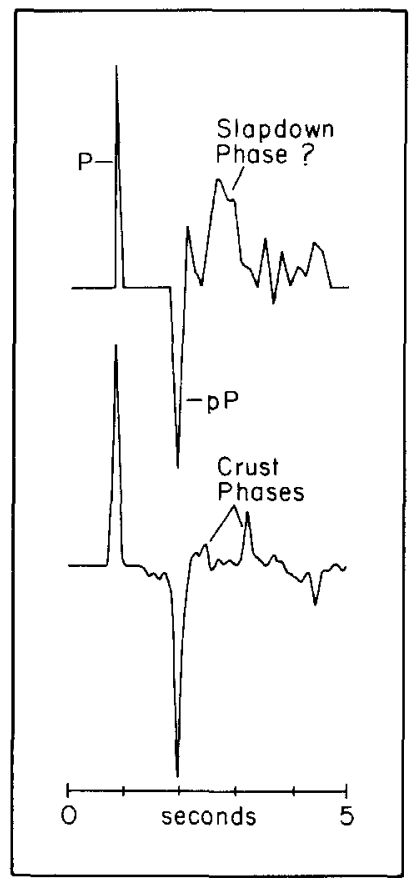

FrG. 8. Top, Cannikin crustal impulse response determined by homomorphic deconvolution (Bakun and Johnson, 1973). Bottom, lightly filtered Haskell response of crust II. The $P-p P$ times agree closely.

The results are given in Table 1 . The short-period amplitudes, which relate to Richter magnitude, increase by a factor of about 6 through the events. We wish to determine how much of this increase is actually due to increase in elastic radius and how much may be due to dynamic effects. The short-period to long-period amplitude ratio is plotted as a function of normalized short-period amplitude in Figure 9. The table and the figure show that as yield increases from 0.7 to $2.7 \mathrm{Mt}$, the short-period to long-period ratio holds constant or perhaps increases slightly. It then drops dramatically for the largest Novaya Zemlya blast (3.2 Mt) and for Cannikin $(<5$ $\mathrm{Mt}$ ). Figure 9 also shows the predicted trajectories of the short-period to long-period ratios as the parameters of the source model are varied over a reasonable range. As the depth or $p P$ delay time is increased from 0.4 to $1.4 \mathrm{sec}$, corresponding to increasing size, the ratio decreases substantially. As the value of $k$ is decreased from 10 to $2 \mathrm{sec}^{-1}$, also corresponding to increasing size, the ratio again decreases. However, if the amount of overshoot increases from $B=2$ to $B=10$, the short- 
period to long-period ratio increases. The short-period record is sensitive to the peak value of the time function while the long period is sensitive to the long-time limit. The theoretical curves are placed arbitrarily on the abscissa of Figure 9 which

TABLE 1

Relative Amplitude Data

\begin{tabular}{rccc}
\multicolumn{1}{c}{ Event } & $\begin{array}{c}\text { Yield } \\
\text { (kt) }\end{array}$ & $\begin{array}{c}\text { Normalized } \\
\text { Short-Period } \\
\text { Amplitude }\end{array}$ & $\begin{array}{c}\text { Short-Period/ } \\
\text { Long-Period } \\
\text { Amplitude } \\
\text { Ratio }\end{array}$ \\
\hline \multicolumn{5}{c}{ Novaya Zemlya } \\
$8 / 29 / 74$ & 870 & 1.0 & 1.0 \\
$8 / 23 / 75$ & 550 & 1.1 & 1.3 \\
$10 / 27 / 66$ & 770 & 1.1 & 1.2 \\
$10 / 21 / 75$ & 700 & 1.5 & 1.3 \\
$10 / 18 / 75$ & 1400 & 1.6 & 1.1 \\
$9 / 27 / 71$ & 770 & 2.1 & 1.3 \\
$10 / 27 / 73$ & 3200 & 3.0 & 0.68 \\
$11 / 2 / 74$ & 1600 & 3.5 & 1.6 \\
$10 / 14 / 70$ & 2100 & 3.7 & 1.7 \\
$9 / 12 / 73$ & 2700 & 5.5 & 1.7 \\
& Cannikin & \\
$11 / 6 / 71$ & $<5000$ & 2.1 & 0.41 \\
\hline
\end{tabular}

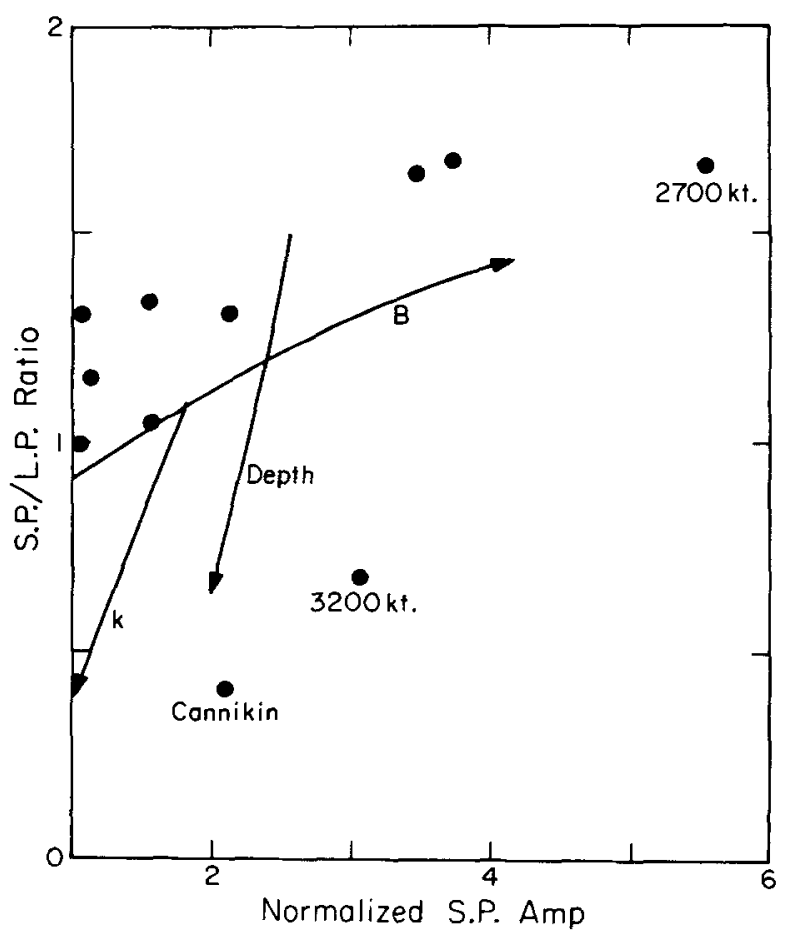

FrG. 9. Observed short-period to long-period ratios as a function of normalized short-period amplitude The curves show the theoretical trajectories taken by the amplitudes as the source parameters are varied. The arrows show the direction of increasing yield.

corresponds to an arbitrary choice of $\psi(\infty)$. The data and theoretical curves in Figure 9 indicate that as bomb size increases, the amount of overshoot increases along with the elastic radius. The increase in seismic magnitude is due to both static and dynamic effects. For the largest two events the effect of source depth dominates and 
the short-period to long-period ratio drops to a low level. It could also be that the largest bombs were conservatively overburied. Since the short-period amplitude depends on so many factors besides $\psi(\infty)$, it is no mystery that it has proved difficult to relate seismic magnitude to yield.

\section{Tectonic Release}

All of the parameters which are necessary to describe the body-wave forms from a pure explosive source have now been considered. However, to complete the

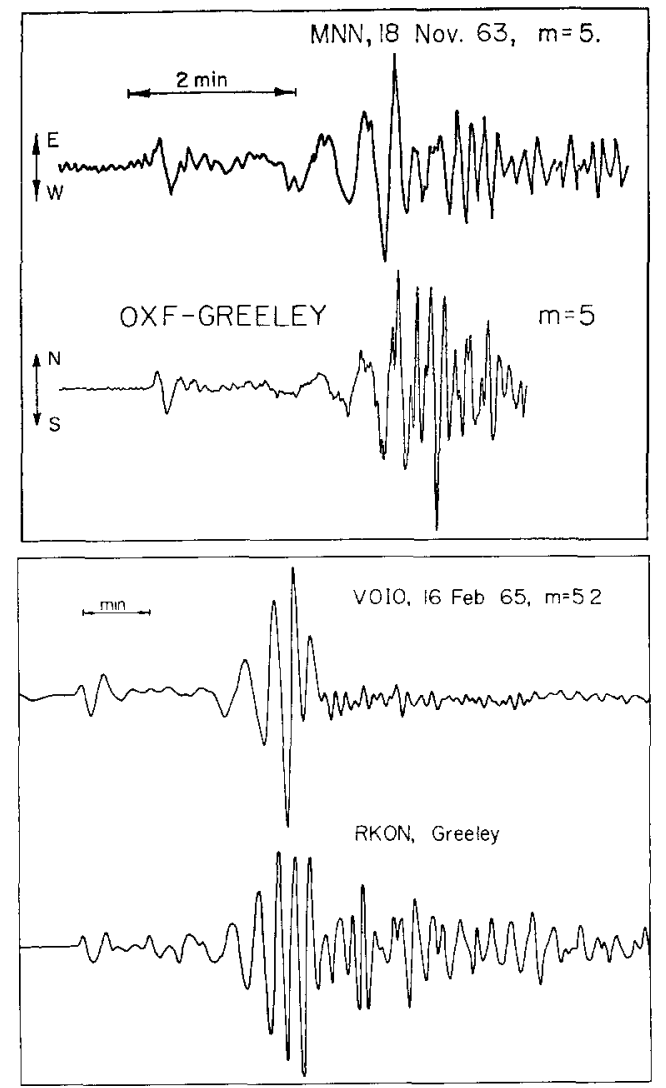

Frg. 10. Comparison of $S H$ records of earthquakes to the $S H$ records of NTS event Greeley. The body waves, in particular, show that there is not a large difference between normal earthquakes and the tectonic release from explosions.

discussion it is necessary to consider the effect of tectonic strain release on the wave forms. Studies of explosion generated surface waves have already led to reliable estimates of the relative size of the tectonic events typically associated with explosions. Toksöz and Kehrer (1972) have provided a list of the surface-wave measurements of tectonic event sizes for a number of NTS and Amchitka explosions. They make use of a parameter $F$ which denotes the ratio of the dc strength of the double-couple source to the dc strength of the explosion. The measured $F$ values for 23 events range from 0 to 3.2 with an average value of 0.78 . The value for Cannikin in particular is listed as 0.6 . In this section, we will attempt to show how tectonic strain release in this magnitude range will affect body-wave forms.

The tectonic release effects can be included in the formalism by simply adding in a double couple (Bache, 1976). It is also possible to add in higher order polar terms 
though this is generally not necessary (Bache and Harkrider, 1976). A summary of the evidence that the tectonic release primarily originates from triggered earthquakes which can be modeled as simple double couples was given by Tsai and Aki (1971). Some additional evidence illustrating that tectonic release is equivalent to an earthquake is presented in Figure 10. The figure compares $S H$ components of motion for the NTS event Greeley with the $S H$ components of earthquakes at the same range. MNN and OXF are WWSSN, and VOIO and RKON are LRSM stations. The $S H$ waves for the explosion and the earthquake are so remarkably similar that it would be impossible to distinguish the two. Therefore, it seems very reasonable to model the tectonic release just as one would an earthquake.

Figures 11 and 12 show short- and long-period $P$-wave synthetics for explosive sources combined with double-couple sources of varying strength. The reduced

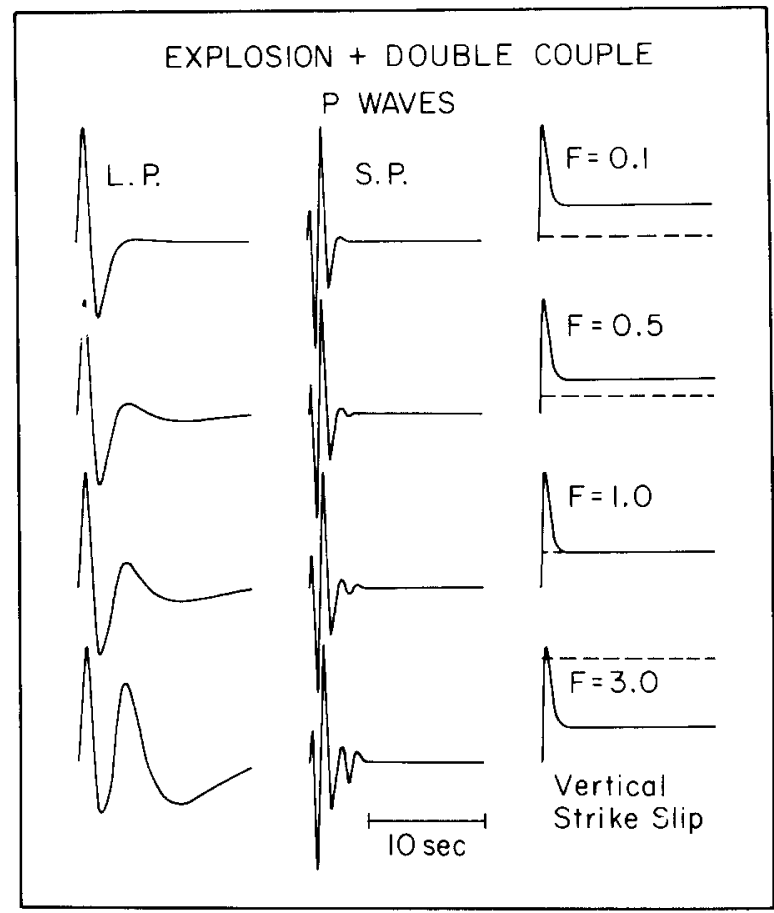

FIG. 11. Short- and long-period records for an explosion plus a vertical strike-slip double-couple point force. The RDP's for the explosive sources are shown on the right. The parameter $F$ is the ratio of the long-period level of the earthquake to the long-period level of the bomb.

displacement potentials for the explosive sources are shown in the right columns of the figures with the dc level of the earthquake indicated as a dashed line. The double couple was placed $1 \mathrm{~km}$ beneath the explosion in a half-space. The synthetics were computed for a ray parameter of 0.06 which corresponds to an epicentral distance of about $60^{\circ}$. The fault plane of the double couple in Figure 11 was vertical strike slip and in Figure 12 it was a $45^{\circ}$ dipping dip slip. The synthetics were computed for the maximum of the $P$-wave azimuthal radiation pattern. In neither case does the tectonic release have a large effect on the wave forms until $F$ is larger than about one. Bache (1976) found a similar result for LRSM short-period $P$ waves. It should be noted that in those cases where the $F$ factor is purported to be larger than one, the body waves should clearly show some effects. The dipping dip-slip double couple radiates $P$ waves to teleseismic distances more efficiently than the 
vertical strike slip, so the effects of tectonic release are more evident in this case. The long periods appear to be somewhat more sensitive to the double couple than the short periods. The most pronounced effects of the tectonic component occur in

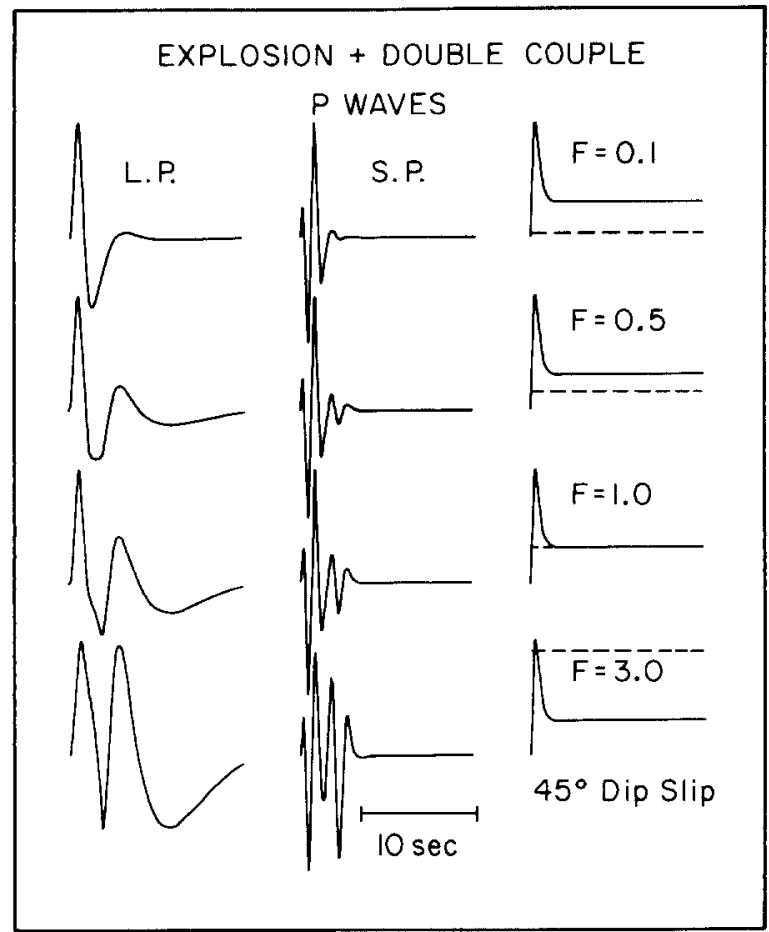

Fig. 12. Short- and long-period records for an explosion plus a dipping dip-slip double-couple point force. The RDP's for the explosive sources are shown on the right. The parameter $F$ is the ratio of the long-period level of the earthquake to the long-period level of the bomb.

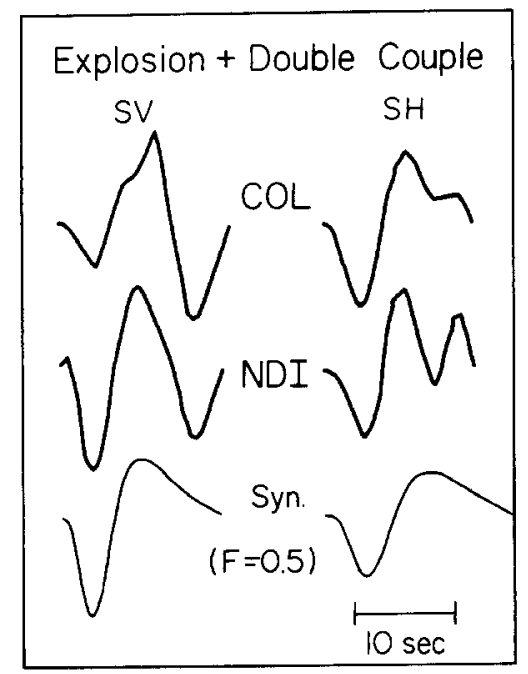

Fig. 13. Observed $S V$ and $S H$ records at 2 stations for the September 12, 1973 Novaya Zemlya blast. Bottom, synthetic records computed for a vertical strike-slip tectonic event with an $F$ factor of only 0.5 . The synthetic $S H$ is already almost large enough to match the observed $S H$ amplitudes.

the second peak of the long-period instrument just as did the source crust reverberations. It is very probable that the two effects combined to produce the often observed structure in this part of the long-period records. 
An important extension of this investigation can be made by calculating the effect of tectonic release of $S V$ body waves. The shear waves from bombs could, in principle, be as useful as the $P$ waves in studies of the Earth's structure (Hart, 1975). However, the studies of Hirasawa (1971) and of R. Butler (personal communication) have shown that the $p S$ phase from explosions is very unstable. The direction of first motion does not always appear to be consistent with an explosive source. This instability can be reasonably explained within the framework of tectonic release.

The observed $S V$ and $S H$ components of motion at WWSSN stations NDI and COL for the September 12, 1973 Novaya Zemlya explosion are show in Figure 13. The stations were naturally rotated so the components did not have to be separated numerically. The $S H$ wave appears to be almost as large as the $S V$. This explains why the sense of first motion is variable when the two components are mixed. At the bottom of the figure are synthetics computed assuming an $F$ factor for a tectonic event of only 0.5 . The calculation assumes a strike-slip source and a maximum for the azimuthal radiation pattern. The $S H$ component is already large enough to explain the data. Comparison with Figures 11 and 12 shows that at this level of tectonic release there is no significant effect on the $P$ waves. The large relative size of the tectonic release $S H$ wave with respect to the explosive $p S$ wave has three causes. First, the $p S$ phase has a conversion coefficient of only 0.6 at the free surface, so it is smaller than direct $P$ to begin with. Second, the double couple radiates $\left(V_{p} /\right.$ $\left.V_{s}\right)^{2}$ higher amplitude shear waves than compressional. Finally, the $\mathrm{SH}$ wave from the tectonic event contains two phases, $S$ and $s S$, which can interfere constructively to give a factor of 2 in $S H$ amplification. From this, we conclude that $S V$ waves from bombs will not be useful tools for probing the Earth unless events can be found with no tectonic release or unless the tectonic release can be accurately modeled.

\section{ConClusions}

The final results of this study are that short-period and long-period body-wave form data from explosions in the 0.3 to $5 \mathrm{Mt}$ range can all be modeled with a von Seggern and Blandford time function. The time scaling parameter $k$ ranges from about 3 to $11 \mathrm{sec}^{-1}$, and the overshoot parameter $B$ ranges from about 2 to 7 . No systematic variation of the parameters with yield can be resolved over this large range of yields even with short-period records. The source depth plays the most important role in determining the wave shape. The values determined for $k$ correlate well with those found by Haskell (1967) and von Seggern and Blandford (1972), who found values of 17 to $32 \mathrm{sec}^{-1}$ for $5 \mathrm{kt}$ explosions in various media. Theoretically, $k$ should scale as the inverse cube root of the yield, so they would predict that for 1Mt blasts, $k$ should range from about 3 to 6 . The long-period wave-form data, the deconvolution study, and the short-period to long-period amplitude ratios all indicated that the time function had to have a substantial overshoot. The range of values of $B$ corresponds to long time levels of the reduced displacement potential which are $\frac{2}{3}$ to $\frac{1}{4}$ of the maximum value. Very comparable results were reported by Aki et al. (1974) from close-in observations and by Bache (1976) from numerical calculations. The strong influence of source depth on both body waves and surface waves was previously noted by Helmberger and Harkrider (1972) who suggested the use of source depth as a discriminant.

The simple model of a point dilatational source buried in an elastic layered halfspace has proved to be very successful in modeling all of the teleseismic body-wave data. There appears to be no need to invoke nonlinear processes in the source region such as slapdown phases. The only possible exceptions would be the decreasing of 
the velocity in the layers above the source and, of course, tectonic release. Tectonic release, however, can also be modeled with a point source and an elastic half-space. The synthetic seismogram calculations presented here have shown that tectonic release will not generally influence the $P$ waves from bombs unless the $F$ factor exceeds 1 . If it is larger than this the tectonic release should be especially evident in the long-period records. The $p S$ phases from bombs are much more sensitive to the tectonic release than the $P$ waves. Their unstable properties at teleseismic distances can easily be explained with a moderately sized tectonic release event.

This study, though it has involved many quantitative methods, has relied only on qualitative measures of goodness of fit. This precludes strong statements concerning the resolution and tradeoffs of the various source parameters. Figures 4 and 5 did show that the source depth can be much more accurately determined from the data than the time function parameters $k$ or $B$. The resolution of the depth depends strongly on the ease with which $p P$ can be identified in the wave form. In a very good situation such as for Cannikin in Figure 6, there is an apparent variation of relative $P$ to $p P$ arrival time of only $0.2 \mathrm{sec}$ reflecting an uncertainty of $400 \mathrm{~m}$ in depth. This, of course, depends completely on the assumed crustal model.

\section{ACKNOWLEDGMENTS}

This research was supported by the Advanced Research Projects Agency of the Department of Defense and was monitored by the Air Force Office of Scientific Research under Contract F49620-77-C-0022.

\section{REFERENCES}

Aki, K., M. Bouchon, and P. Reasenberg (1974). Seismic source function of an underground nuclear explosion, Bull. Seism. Soc. Am. 64, 131-148.

Anderson, D. L. and R. S. Hart (1978). Attenuation models of the earth, Phys. Earth Planet Interiors 16, 289-306.

Bache, T. C. (1976). The effect of tectonic stress release on explosion $P$-wave signatures, Bull. Seism. Soc. Am. 66, 1441-1537.

Bache, T. C. and D. G. Harkrider (1976). The body waves due to a general seismic source in a layered earth model: 1. Formulation of the theory, Bull. Seism. Soc. Am. 66, 1805-1819.

Bakun, W. H. and L. R. Johnson (1973). The deconvolution of teleseismic $P$ waves from explosions Milrow and Cannikin, Geophys. J. 34, 321-342.

Burdick, L. J. and G. R. Mellman (1976). Inversion of the body waves of the Borrego Mountain earthquake to the source mechanism, Bull. Seism. Soc. Am.66, 1485-1499.

Dahlman, O. and H. Israelson (1977). Monitoring Underground Nuclear Explosions, Elsevier Scientific Publishing Co., Amsterdam, 440 pp.

Engdahl, E. R. (1972). Seismic effects of the Milrow and Cannikin nuclear explosions, Bull. Seism. Soc. Am. 62, 1411-1424.

Frasier, C. W. (1972), Observations of $p P$ in the short period phases of NTS explosions recorded at Norway, Geophys. J. 31, 99-109.

Fuchs, K. (1966). The transfer function for $P$-waves for a system consisting of a point source in a layered medium, Bull. Seism. Soc. Am. 56, 75-108.

Haskell, N. A. (1967). Analytic approximation for the elastic radiation from a contained underground explosion, J. Geophys. Res. 72, 2583-2587.

Harkrider, D. G. (1964). Surface waves in multilayered elastic media I. Rayleigh and Love waves from buried sources in a multilayered elastic half-space, Bull. Seism. Soc. Am. 54, 627-679.

Hart, R. S. (1975). Shear velocity in the lower mantle from explosion data, J. Geophys. Res. 80, 48894894.

Helmberger, D. V. (1973). On the structure of the low velocity zone, Geophys. J. 34, 251-263.

Helmberger, D. V. and D. G. Harkrider (1972). Seismic source descriptions of underground explosions and a depth discriminate, Geophys. J. 31, 45-66.

Helmberger, D. V. and R. Wiggins (1971). Upper mantle structure of the midwestern United States, $J$. Geophys. Res. 76, 3229-3245.

Hirasawa, T. (1971). Radiation patterns of $S$ waves from nuclear explosions, J. Geophys. Res. 76, 64406454 . 
Langston, C. A. and D. V. Helmberger (1975). A procedure for modeling shallow dislocation sources, Geophys. J. 42, 117-130.

Molnar, P. (1971). $P$ wave spectra from underground nuclear explosions, Geophys. J. 23, 273-287.

Mueller, R. A. and J. R. Murphy (1971). Seismic characteristics of underground nuclear detonations; Part I, Seismic scaling law of underground detonations, Bull. Seism. Soc. Am. 61, 1675-1692.

Springer, D. L. (1974). Secondary sources of seismic waves from underground nuclear explosions, Bull. Seism. Soc. Am. 64, 581-594.

Toksöz, M. N., A. Ben-Menahem, and D. G. Harkrider (1964). Determination of source parameters of explosions and earthquakes by amplitude equalization of seismic surface waves, J. Geophys. Res. 69, 4355-4366.

Toksöz, M. N. and H. H. Kehrer (1972). Tectonic strain release characteristics of Cannikin, Bull. Seism. Soc. Am. 62, 1425-1438.

Tsai, Y. and K. Aki (1971). Amplitude spectra of surface waves from small earthquakes and underground nuclear explosions, J. Geophys. Res. 76, 3940-3952.

von Seggern, D. and R. Blandford (1972). Source time functions and spectra for underground nuclear explosions, Geophys. J. 31, 83-97.

Willis, D. E., G. D. George, K. G. Poetzl, C. E. Saltzer, A. F. Shakal, R. D. Torfin, T. L. Woodzick, and C. Wolosin (1972). Seismological aspects of the Cannikin nuclear explosion, Bull. Seism. Soc. Am. 62, 1377-1396.

Seismological laboratory

Division of Geological and Planetary Sciences

Caimfornia Institute of Technology

Pasadena, California 91125

Contribution No. 3158

Manuscript received September 29, 1978 